\title{
EWSR1/PATZ1 Fusion Gene Short Isoform
}

National Cancer Institute

\section{Source}

National Cancer Institute. EWSR1/PATZ1 Fusion Gene Short Isoform. NCI Thesaurus.

Code C99239.

A fusion gene $(\sim 1.9 \mathrm{~kb})$ that results from a chromosomal inversion inv(22)(q12q12) which fuses the first eight exons of the EWSR1 gene with the PATZ1 gene. This rearrang ement is associated with Ewing tumor/peripheral primitive neuroectodermal tumor. 